Proceedings

\title{
Integrating Einsteinian physics in the year 7 Australian Science Curriculum: What are the challenges in design and implemen- tation?
}

Shon Shachar Boublil

Citation: Lastname, F.; Lastname, F.; Lastname, F. Title. Proceedings 2021, 68, x. https://doi.org/10.3390/xxxxx

Published: date

Publisher's Note: MDPI stays neutral with regard to jurisdictional claims in published maps and institutional affiliations.

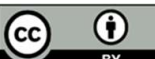

Copyright: @ 2021 by the authors. Submitted for possible open access publication under the terms and conditions of the Creative Commons Attribution (CC BY) license (http://creativecommons.org/licenses/b y/4.0/).

\begin{abstract}
Integrating Einsteinian physics into school curricula has become a challenge for researchers. This process involves choosing appropriate disciplinary knowledge to be accommodated in the curriculum and researching ways to illustrate how teachers can use this knowledge in their classrooms. The Einstein-first project research is centered on building a new curriculum on Einsteinian

physics (space, time, geometry, gravity) in Western Australia and on teacher's ability to embrace this modern paradigm and enhance their scientific and didactical knowledge. My research is designed to trial a learning progression of Einsteinian concepts within an overall curriculum structure for year 7. Many concepts related to Einstein's theory of gravity will be included in association to the existing year 7 curriculum in Australia. I will identify the primary challenges in design and implementation, which helps organize appropriate teacher professional learning to understand and teach Einsteinian physics concepts. A series of 14 physics lessons were developed for the Year 7 Science curriculum in Western Australia. The first three lessons introduce concepts of measurement, straight lines, Geometry, space (curved), time, nature of spacetime. The concept of velocity, terminal velocity, acceleration, inertia, and mass are then developed. Students then learn about Einstein's conception of gravity through the analysis of free-falling bodies and thought experiments. They use the spacetime simulator to investigate topics of general relativity, the attractional force between masses and orbits in our Solar System. The learning progression ends with introducing black holes and gravitational waves.
\end{abstract}

\section{What is space? (laser experiment)}

This lesson starts with the question "What is space?". A brief PowerPoint overview of the history of geometry from Euclid to Newton to Gauss to Einstein. This includes Gauss's first testing of the shape of space, and eclipse experiments that proved that space is curved. This brief introduction finishes with the John Wheeler's aphorism for Einstein's theory: matter tells spacetime how to curve, spacetime tells matter how to move. A student writes this on the white board and class repeats it aloud. Teacher explains that the next lessons will give them an understanding this aphorism.

To measure the shape of space we

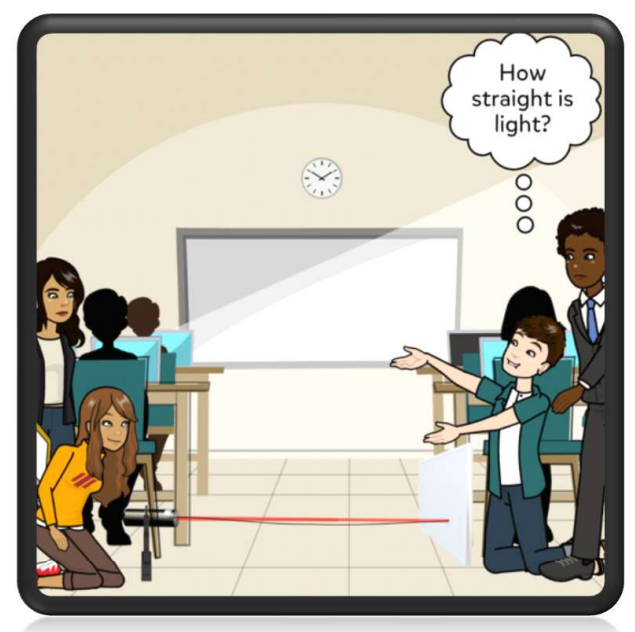
need geometry. Geometry itself needs the concept of straight lines. The activity investigates concepts of straightness, space, dimensions and light. Every group of students will use a stretched string, ruler and laser to measure the deviation from straightness using visual sighting to the straight path of light. They will measure how much the string sagged in relation to light. The lesson finishes with a brainstorm in small groups where each team 
spends a few minutes to answer the question "what is a straight line". Then through teacher-led discussion, two definitions emerge, the shortest path and the path of light.

\section{Geometry of space (wok experiment)}

We revise the three key ideas from lesson 1: space is curved, geometry allows the shape of space to be measured, straight lines are the shortest path between two points and the path taken by light beams.

Dimensions of space: Teacher briefly introduces concepts of 1D, 2D, 3D and 4D spaces, with time as the $4^{\text {th }}$ dimension. Remember the aphorism: it says spacetime.

Gauss was the first to question the shape of 3-dimensional space. Let's try his experiment: geometry in 3D

Class demonstration: three students at-

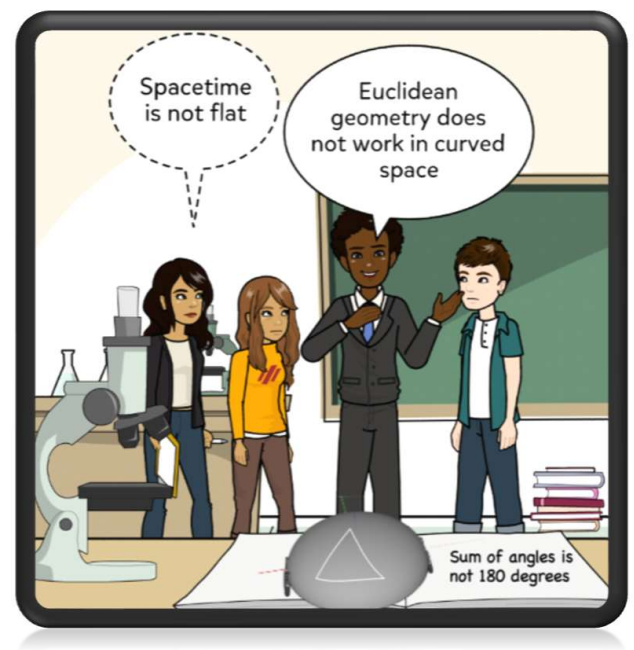
tempt to reproduce Gauss's light beam triangles experiment in 3D using mirrors and laser pointers. NOT TIME FOR OUTSIDE

The demo shows the difficulties of 3D geometry, so we settle for geometry in 2D.

Our tools : the spacetime simulator and the wok. We will use only woks today. In the activity, students will investigate triangles on a curved wok. They will survey straight lines, verify their straightness, and measure the angle sum of large and small triangles on the curved 2D surface. They will make graphs of the sum of angles vs. perimeter and see that curved space has angle sum's greater than 180 degrees.

\section{Discovering spacetime (Stone age running race)}

Revise the key idea so far: spacetime is curved and the shape of space can be measured. Later we will discover warped time too. But how do we measure space and time? This lesson aims to give students insight into: how speed links space with time. It starts by investigating how measuring space and time might have begun in the stone age -the paleolithic period. We ask students to brainstorm how you could measure the sprinting speed of a runner before rulers and clocks were invented. Small groups brainstorm and report back. Then teacher led class discussion converges on the use of heart beats as counters for paleolithic seconds and

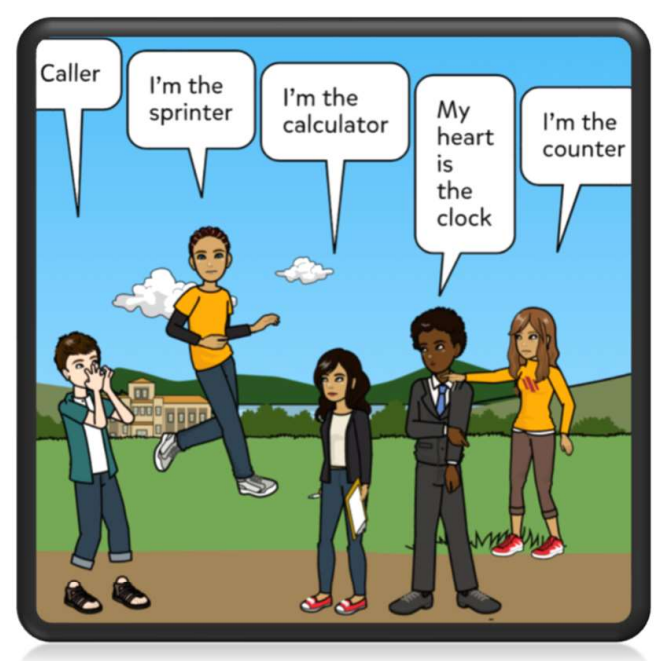
paces as counters for paleolithic meters.

The activity consists of 50-pace time trials in 5-person groups. Groups measure 50 paces and mark start and finish points. One person is the heartbeat clock, one is the pulse counter, one the runner, one calls start/finish, and the last calculates the speed. Each group records time as the number of heartbeats for 50 paces, and calculates average speed such as " 5 paces per heartbeat". They report results to the teacher. 
Teacher asks each group to brainstorm two ideas: "What is wrong with paleolithic units?" and then . "Imagine and describe aliens with completely bodies: how would their units differ from our units?" Many answers: hearts not steady, people different sizes. Aliens with fast hears, short legs! Conclusion: our units arose because accidental properties of our bodies: our hearts beat about once per second and our legs are about a meter long. Paleolithic units were refined to our modern seconds and meters over thousands of years. Students in small groups are then asked to brainstorm and come up with six statements involving distance and measurements such as "the supermarket is 5 minutes down the road". As groups report, teacher asks "what speed did you assume?" for each answer. Students recognise need to Identify the speed assumed for each case.

Does the universe have a speed everyone could agree on? Tease out the key answer: the speed of light is always the same: it is the speed limit of the universe. It provides a universal link between space and time, so instead of two arbitrary quantities, we have only one: we can measure space and time in units of time. Ask students to give examples of measuring distances in light travel time.

\section{Spacetime and motion (Balloon experiment)}

Revise lesson 3 by reviewing light speed measurements of distance: how far to the moon, to the Sun, to Alpha Centauri, to the centre of the Milky Way. How do you control a Mars rover when it is 15 minutes away?

Understanding limiting speeds: This lesson begins with students in groups brainstorming, googling and listing speed records: running, swimming, racing cars, fastest rocket, fastest jet plane, fastest sailing ship, fastest mammal etc. For each item, students must guess what limits the speed.(eg fuel supply, air resistance, blood flow) Teacher led discussion creates a list, with likely limiting causes given for each. This motivates a later discussion of how the universal

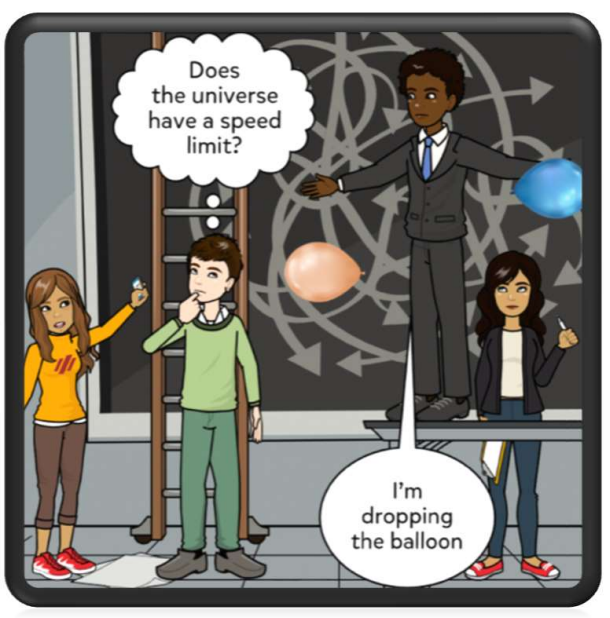
speed limit c, is maintained.

The activity consists of small groups timing the dropping three balloons containing 5-50 g of water when dropped from ceiling to floor. A phone records each fall. Students plot spacetime graphs of height vs. time (each only two points) and then brainstorm what the line joining the start and finish points should look like. Teacher led discussion on the guessed shape of the curves between start and finish. Teacher questioning: will balloons continue to go faster? Did they accelerate? How can we be sure? What was the initial speed? Next lesson students will determine the true shape of the curve. Guide discussion to identify a) slope of graph is speed, b) initial speed is zero c) was it still accelerating when it hit the floor. Each group to predict true shape of curve, for comparing in the next lesson.

Teacher led discussion on how air resistance affects free fall leads to the Brian Cox video of ball and feather falling in a vacuum chamber. If you could fall forever would you reach infinite speed?

Teacher led discussion: speed limit of the universe. Project star shot: things get heavier as they go faster. Show graph mass vs. speed. That is why no rocket could ever reach light speed...it would be infinitely heavy!

Worksheet: Distances measured with time at light speed. Use light travel time to describe distance to Alpha Centauri, center of the Milky Way, to the most distant galaxies.

\section{Terminal speed of a balloon (computer lab)}


This lesson reinforces the previous one and specifically identifies the two phases of motion: acceleration and terminal speed.

This lesson will make use of the experiment and results from the previous lesson. Students use a digital tool to analyse video frames of falling balloons. Remind students that they have seen that the weight of the balloons did affect their free fall because of air resistance. In this class we will see how the balloon reaches a terminal speed.

Each Team should upload their 3 balloon videos to online to https://jst.lucademian.com/. Teacher demonstrates how to use

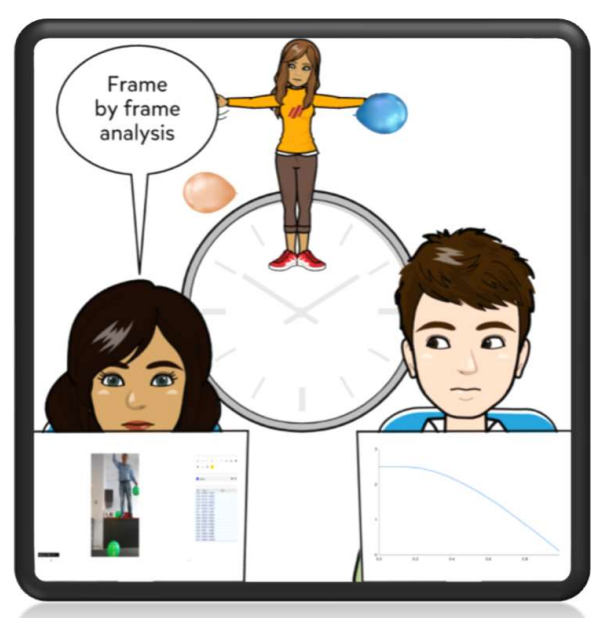
the software. Students follow the guidelines of tracking the balloon. All the motion data is then copied and pasted on to Google sheets in order to create a graph. The class finishes with a teacher led discussion about how balloons accelerated and then reached a speed limit. Air and gravity are enforcing speed limit to this balloon.

\section{Thinking about Inertia, Mass, Momentum and Forces}

This lesson starts with teacher introducing images of medieval battering rams for breaking down castle walls. Then teacher asks students to brainstorm the following thought experiment on inertia: pushing the teacher's car in a flat car park. Small groups brainstorm all the things that could go wrong? Groups report possible scenarios: speeding up really slowly, but getting faster and faster as people keep pushing. Once started it is hard to stop. It crosses the car park and hits a wall, smashing the back of the car or maybe it bashes into the principal's car which bounces into another car.

Teacher led question session: what are the key concepts behind the car pushing thought experiment? Guide discussion towards the following: a) Mass: the amount of matter in an object. b) Inertia: resistance to changes of motion through space. Inertia tells us about how mass is connected to space. c) Momentum: Mass x speed. Momentum measured ability to do damage. Make two lists: things that have high momentum because of speed, and those that have momentum mostly from mass, and those that have both (eg planet Earth).

Teacher optional fun demo: glass bottle, coin and $\$ 50$ note: remove the note without disturbing the coin.

Teacher led discussion: how do you measure mass without the help of gravity? How do astronauts weigh themselves when they are weightless?

Activity: bathroom scales for the ISS: using rulers and blutak to weigh toy astronauts. By filming the oscillations of a bendy ruler with known masses, students make a graph of period vs. mass for 4 mass values. Then use their graph to estimate the mass of the toy astronaut. Confirm result with spring balance. 
Small groups brainstorm: Why do massive objects fall the same as low mass objects. Groups report suggestions to teacher who records ideas on whiteboard. Next lesson we will find out how Einstein answered this question.

\section{Einstein's Happiest Thought: Experiments in Freefall}

Review concepts of mass, inertia, and momentum. Changing momentum means acceleration and that needs forces: that is why battering rams can smash castles

PPT: The story of Einstein's discovery of gravity. a) Einstein discovers the speed limit of the universe in 1905 . b) Newton's law of gravity says there are instantaneous forces between things like the Earth and the Sun that makes planets go in circles around the Sun. If the Sun suddenly disappeared what would happen to the Earth's orbit. Newton would say it would instantly start moving in a straight line: Einstein said that was impossible: it would take 8 minutes be-

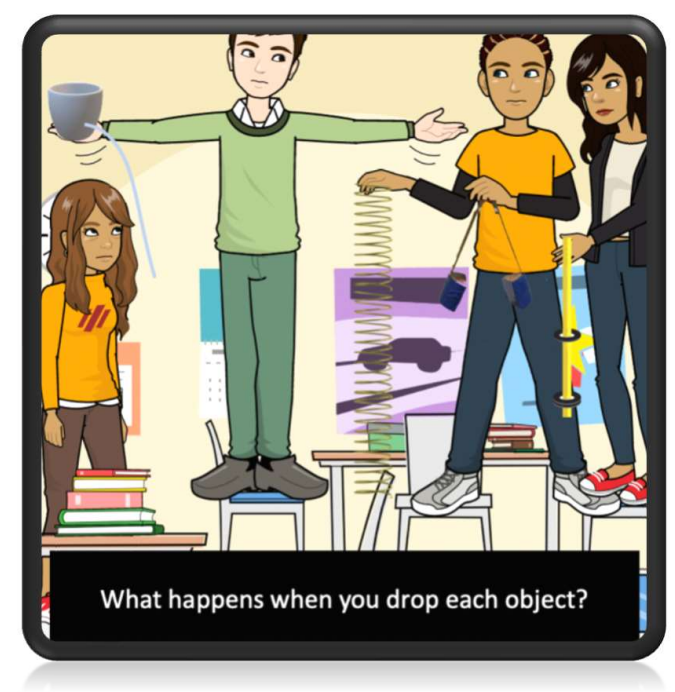
cause nothing can go faster than light. c) 1907: imagine Einstein joyriding in those new things called cars: he enjoyed acceleration. That year Einstein was sitting in a chair at the Patent Office: he jumped up with excitement: he later said this was the happiest thought of his life - gravity is indistinguishable from acceleration. We constantly feel acceleration: the atoms in the Earth exert forces on our bodies just like a car seat prevents us from falling out the back of a car.

The car seat lets us feel the car's acceleration and the Earth lets us feel the acceleration we call gravity. Free fall is any motion where the Earth is not holding you up.

Activity: What happens to things in free fall?

Small groups (about 4) will work successively with three objects and a worksheet containing three boxes: 'predict, observe, explain'. Teacher controls class, first to predict what will happen when object is a)dropped b) thrown upwards. When all predictions have been made, ask all groups to try both dropping and throwing upwards, then record observations, then brainstorm to complete the explanation. Repeat three times, one for each activity.

Tape measure masses: no gravity in free fall

Leaky bottles: no gravity to force the water out

Magnetic beads: no gravity in free fall but magnetism is unaltered.

Teacher led discussion: Groups report what they learnt from each experiment.

Activity: The vanishing Sun. Whole class demo experiment performed by chosen students. Student suspends a stretched slinky with small ball attached to the end to represent the Earth. The slinky spring represents the force of gravity on the Earth from the Sun (the Sun is the hand holding the top of the slinky). If the Sun suddenly ceased to exist, how would the Earth respond. When the Sun vanishes, there is no more force. Ask students to predict what will happen. They observe that the force continues until the release "information" has reached the other end. The Earth continues to move in a circle for 8 more minutes.

\section{Einstein's Thought Experiments: enrichment lesson}


In order to understand gravity, we need to understand things that happen in spacetime. Our understanding of motion and spacetime is important for understanding Einstein's theory of gravity. Remember, gravity will make massive and less massive objects fall the same way, without the presence of air. We start by a group brainstorm where students answer the following questions: What happens to your body when a car accelerates? Teacher-led discussion on these concepts: thought experiments are devices of the imagination. Einstein and Newton were masters in making thought experiments. Teacher-led presentation on gravity: Newton and Galileo knew that

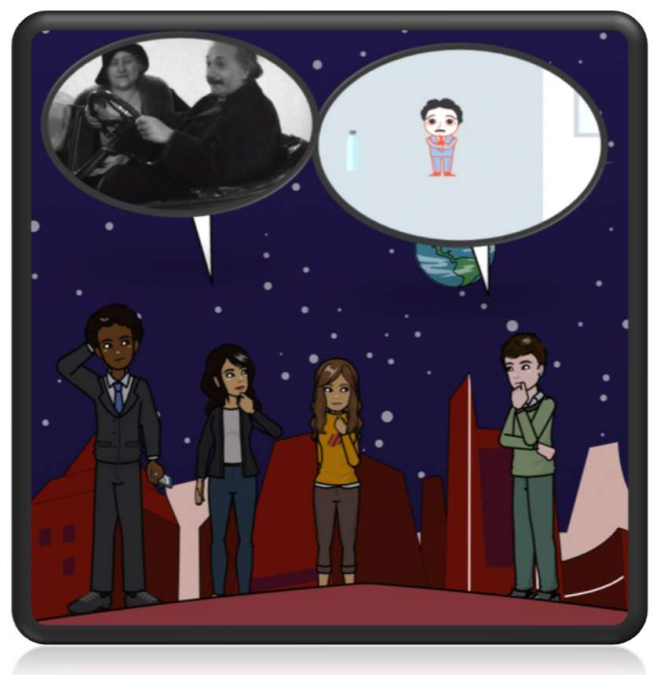
objects with huge inertia fall the same way as objects with small inertia. Both could not explain why that was. Newton's model of gravity needed to be revisited. When Einstein was sitting in a chair at the Patent Office, the happiest thought came to him: "If I fall freely, I will not feel my own weight." This idea was probably inspired by the fact that you are pushed back into the seat of a car when accelerating. Watch a depiction of Einstein's happiest thought.

To properly understand why a person in free fall will not feel their own weight, we will explore how Einstein's thought experiments allowed him to develop our current theory for understanding gravity. Teacher plays the Power Point and targets the initial conceptions of students in relation to Einstein's happiest thought and complete the questions in the worksheet individually. Then make students go into groups or individually and give students time to start working on the questions asked in the worksheet.

\section{Einstein's theory of gravity (measuring spacetime curvature on the spacetime simu- lator)}

In this lesson students will explore Einstein's most famous analogy for explaining gravity. By Measuring the stretching of the spacetime simulator: adding masses to the centre of STS and measuring the distance between two dots. This lesson begins with a PPT about how time depends on height, how the centre of the Earth is more than 2 years younger than the surface, how time runs faster on the space station and time comes to a stop at the surface of a black hole. Brief mention of the best atomic clocks in the world that can easily measure these ef-

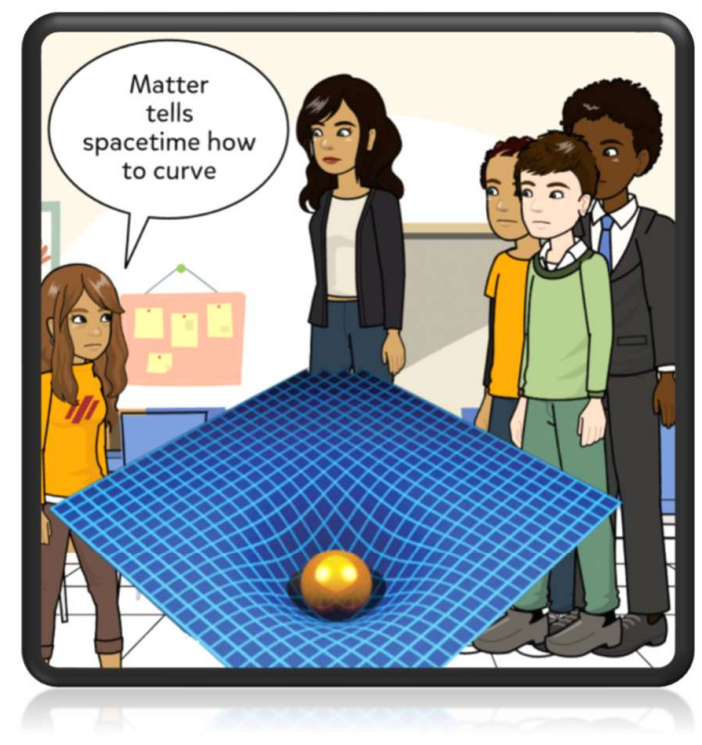
fects. In this lesson students will explore gravity on the spacetime simulator but recognising that the stretching of the lycra sheet represents the stretching of space and time. 
Activity 1: Students measure the stretching of the membrane as central masses are added and interpret it as the stretching of space and time.

Activity 2:The next activity investigates forces on the simulator. Students measure Newton's law of gravity and plot force vs. distance for different masses. They observe that the force depends on the product of the two masses. Finally they consider the two ways that the simulator can be interpreted: one to observe the curvature of space and the other the stretching in time.

\section{Einstein's theory of gravity and light bending (mapping toy photon trajectories)}

Introduce that in our previous lesson, we discussed a new description of gravity that is caused by the presence of matter and spacetime curvature. We are going to investigate how Einstein's theory of gravity predicted the bending of light and explore this phenomenon on the simulator.

Teacher presents that gravity bends light, especially when light approaches massive objects like our Sun. During a solar eclipse, Eddington (Sobral) and Ross (Wallal) measured how light from a star shifted when observed near the

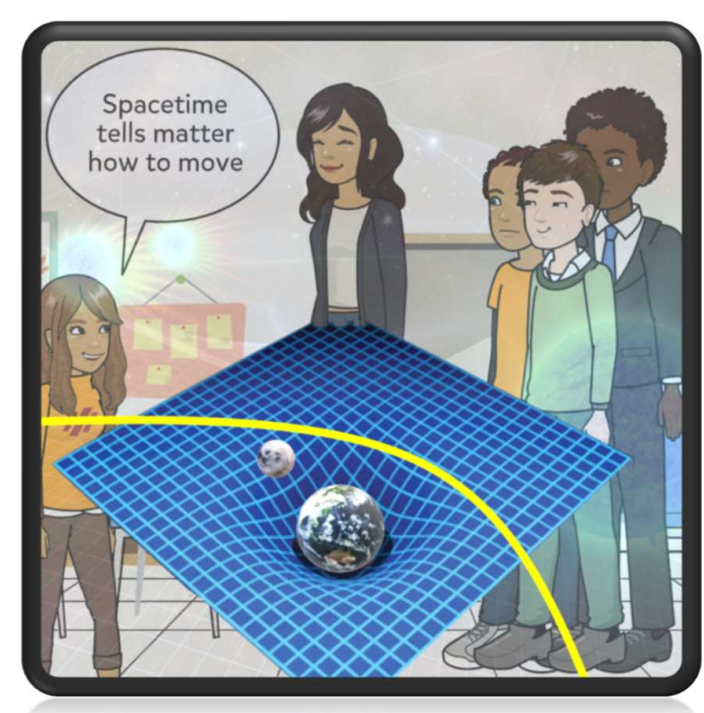
Sun. Two videos about this events are shown. Introduce photographs of gravitational lensing.

Teacher led demonstration and whole class discussion Get the students around the space time simulator. Demonstrate that this pull back car will be used as light travelling in space. This toy photon car on the STS, shows us analogically that light travels in straight lines in a space with no mass, thus no gravity. Place a mass in the center of the and make every group release the pullback car at a specific location on the simulator. (Each group will place a dot on the side of the spacetime simulator). Each group should measure where the car was received. Repeat this process for all the masses and insert findings in the activity sheet.

\section{Orbits and our solar system (three experimental stations to investigate Kepler's laws)}


In this lesson, students will record the trajectories of marbles on the spacetime simulator. Students will get to investigate three activities investigating Kepler's three laws of motion. This will give a historical understanding of how we started to make sense of the motion of planetary objects in our solar system. Instructions to three experimental stations to investigate Kepler's laws will be given. Students will learn how to draw ellipses and understand Kepler's first law. They will quantitatively measure the area swept by a planet in relation to the time it took a planet to travel from one location to another. This will allow them to understand Kepler's $2^{\text {nd }}$ law.

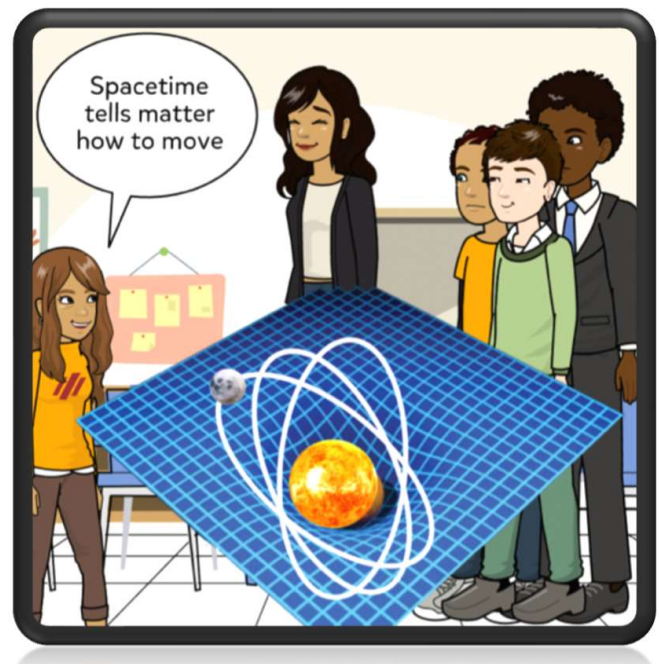

Students time circular orbits for planets like Neptune, Earth and Mercury. Make a rough graph of orbital radius vs. orbital period. Students investigate to see if the STS follows the radius-period relation called Kepler's Law: period squared = orbit radius cubed. Teacher led discussion on the precession of Mercury due to curved space in which geometry does not obey the usual formulae about circles.

\section{Schwarzschild's Outrageous Solution.}

Roleplay: Timeline of discovery: 1905-2017

Students act out the succession of discoveries from 1905 to 2017 that brought us black holes and gravitational waves.

\section{Black holes and Gravitational waves (VR, laser interferometer and soap film inter- ference)}

Introduce that Black holes the most powerful energy machines in the universe and is a place wher spacetime cease to exist. Einstein did not believe the universe could have black holes. Black holes can the size of central city to the size of the solar system. The concept of gravitational waves and the discovery of gravitational waves will be introduced via video two videos. Teacher explains that scientists around the world have found a new way to detect astronomical events in our universe. Scientists have managed to detect the merging of two black holes and two neutron stars using a very precise laser interferometer. Students will first use virtual reality to explore black holes

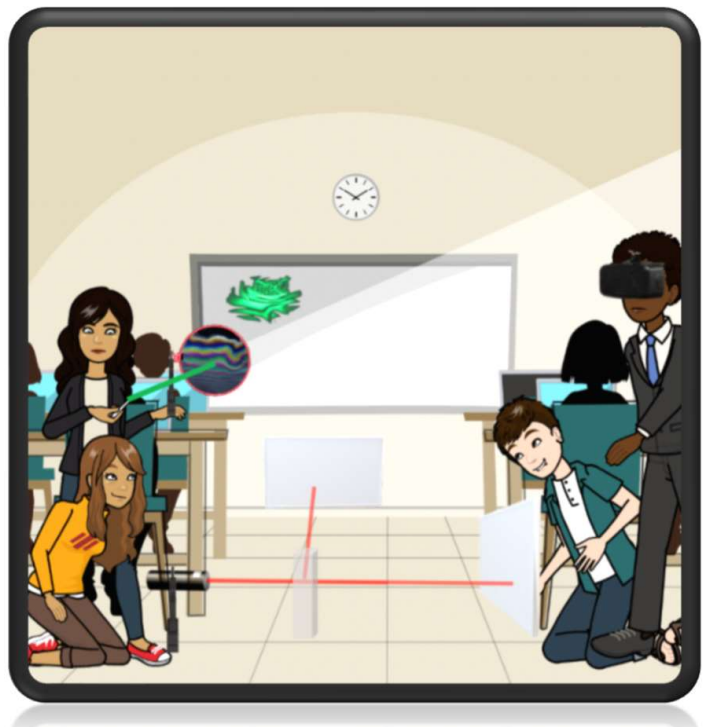
merging and then hands on experiments to understand how gravitational waves are detected. 
A beam splitter experiment will be used. Students will build a toy interferometer and achieve laser beam alignment at long distances and understand how difficult it is to align the beams to hit the two mirrors. A nanometre depiction of interference patterns using lasers and soap film. The teacher will demonstrate the interference patterns and show we are observing reality at the nanometre scale. However at the ligo they are looking at disturbances in space at the $10^{\wedge}-33 \mathrm{~m}$ scale. That is 10000 of the size of a proton. They are able at measuring reality at that scale.

\section{The Mystery of blackholes and the end of spacetime}

End the course with very short videos each followed by brainstorm discussions after each video:

Black holes and neutron stars merging throughout the universe. Students Brainstorm Imagine seeing spacetime like surface of the ocean: what would this image be like? Each group reports 3 ideas Draw pictures if needed.

Black holes in the centres of galaxies Students brainstorm how might we find out if a black hole was approaching the solar system

Hawking ideas of blackholes. Students brainstorm can the universe end up as one big Black hole or could a planet be powered by its own black hole if their sun

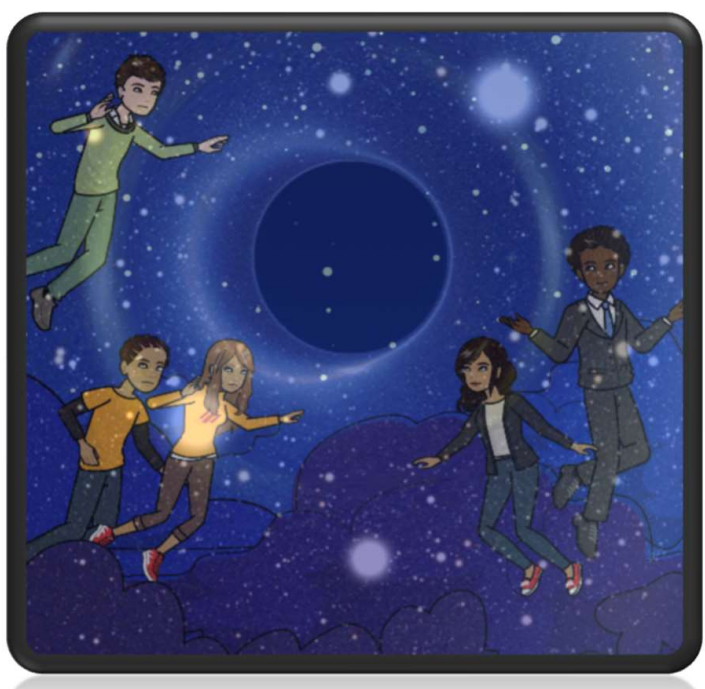
died

Dark matter and energy video. Student brainstorm What is dark matter and energy? How do they know it exists? 